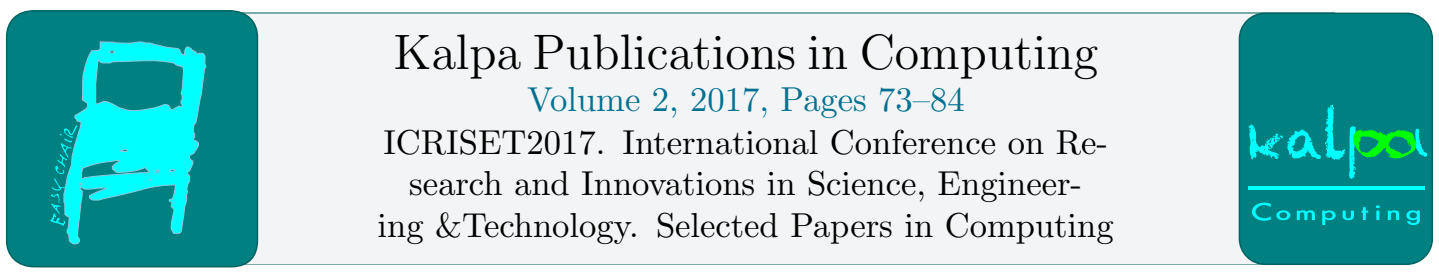

\title{
Combined effect of deformation and slip velocity on the performance of a Ferrofluid squeeze film in infinitely long porous rough rectangular plates
}

\author{
Mukesh Shimpi ${ }^{1}$ and G M Deheri ${ }^{2}$ \\ ${ }^{1}$ Department of Mathematics, Birla Vishvakarma Mahavidyalaya Engineering College, \\ Vallabh Vidyanagar. \\ ${ }^{2}$ Department of Mathematics, Sardar Patel University, Vallabh Vidyanagar. \\ mukesh.shimpi@gmail.com, gm.deherierediffmail.com
}

\begin{abstract}
This investigation aims to study the performance characteristic of a Ferrofluid based squeeze film in infinitely long porous transversely rough rectangular plates considering the effect of deformation and slip velocity. The magnetic fluid flow model of Neuringer and Rosenweig has been used. Beavers - Joseph slip model has been adopted and the stochastic method of Christensen and Tonder has been deployed for roughness. The associated stochastically averaged Reynolds' type equation is solved. The results prove that the Ferrofluid lubrication offers a limited help in reducing the adverse effect of slip velocity, deformation and porosity, even in the case of negatively skewed roughness. However, the situation improves when variance (- ve) occurs and lower to moderate values of deformation is involved. Aside, this study shows that the slip coefficient has to be at the reduced level an improvement of the bearing performance.
\end{abstract}

\section{Introduction}

The squeeze film phenomenon evolves when two lubricated surfaces move towards each other with a velocity called squeeze velocity, in the normal direction, generates a positive pressure, and hence sustains a load. In addition, this process provides a cushioning effect in bearings. The squeeze film phenomenon is widely observed in many applications such as bearings, machine tools, engines, gears, rolling elements and automotive engines etc. It was obtained that the squeeze time increased if a fluid with couple stress was used as the lubricant and among all the shapes with same area, the circular shape gave the maximum squeeze time. [Cameron, 1966] studied the squeeze film lubrication 
between two infinitely long parallel plates. Now a day, it is well known that the roughness of the bearing surfaces has a significant effect on the performance characteristics of a squeeze film bearing system.

The roughness invariably is manifest to be random in character accordingly a stochastic approach has been used to mathematically model the roughness of the bearing surfaces [[Tzeng\&Saibel, 1967] and [Christensen \& Tonder 1969-70]]. Surface roughness affects several functional attributes of parts, such as wear, friction, light reflection, heat transmission, holding a lubricant and coating etc.A number of investigations [[Christensen\&Tonder, 1967-70], [Guha, 1993], [Bhat,1993], [Gupta \& Deheri, 1996], [Prakash\& Gururajan, 1999], [Patel et al., 2014] and [Shimpi \& Deheri, 2014]]analyzed the performance in magnetic fluid based rough bearing systems.

[Patel et al., 2014] studied the effect of non-uniform thickness on the magnetic fluid based squeeze film in rough porous long plates. Further, the variable film thickness supported the magnetization in minimizing the adverse effect of porosity and roughness.

The no-slip boundary condition is the foundation of traditional lubrication theory. As a result, fluid adjacent to a solid boundary has zero velocity as compared to the solid surface. For many practical applications, the no-slip boundary condition may be a good model for predicting the behaviour of fluid. However, the experimental research has shown that for special engineered surfaces the no-slip boundary condition is practically not applicable. Measured velocity profiles put forward that slip usually occurs at the edges. Slip was widely inspected in polymer melts, which is now promising to be an intense field of research due to the advances in micro and Nano-scale technology. Researcher must not forget that the validation of high-order velocity-slip boundary condition is for gas micro flows. It has been found that slip becomes an important factor at the small scales, which makes slip an important Tribological consideration for micro machine technology. If slip surfaces prove feasible, there is great potential for their application in bearings, since it is believed that they can reduce energy losses and increase load carrying capacity $\mathrm{W}$ and hence, bearing performance.

[Murti, 1973] informed that the effects of velocity slip were prominent when the bearing operated at a lower eccentricity ratio and/or the bearing-matrix had a low permeability. [Verma, 1986] considered tangential slip velocity at the porous matrix lubricant interface, while [Agrawal, 1986] discussed no slip condition. [Fortier Alicia \& Salant Richard, 2005] explored theoretically the slip phenomenon to improve the fluid film bearing performance by considering judicious choice of both slip and no-slip bearing surface called heterogeneous pattern. [Shukla \& Deheri, 2014] studied the effect of slip velocity on the performance of a magnetic fluid based squeeze film in porous rough infinitely long parallel plates.

It was known from [Higginson, 1965] that the elastic deformation on the bearing liner on journal bearing performance were not negligible as compared to film thickness.

[Prakash\&Peeken, 1985] investigated the effect of surface roughness and elastic deformations in one-dimensional slider bearing and found the existence of strong interaction between the roughness and elastic deformation. [Schwarz, 2003] presented a new method to calculate the elastic deformation of a sphere on a flat surface considering the influence of short range and long range attraction forces inside and outside the actual contact area. [Chatchai\&Mongko, 2010] proved that the roughness pattern, elastic deformation, power law index significantly affected the characteristics of journal bearing under severe operating conditions. [Shimpi \& Deheri, 2013] studied the combined effect of rotation and bearing deformation on the Ferrofluid based squeeze film in rotating curved transversely rough porous circular plates. The negatively skewed roughness introduced a better performance for a good range of deformation, by suitably choosing the curvature parameters.

Here an endeavor has been made to discuss the combined effect of deformation and slip velocity on the performance characteristics of a Ferrofluid based squeeze film in infinitely long porous transversely rough rectangular plates. 


\section{Analysis}

The geometry and configuration of the bearing system is presented in Figure -1 , where in the upper plate approaches the lower plate normally with uniform velocity $\dot{h}\left(=\frac{d h}{d t}\right)$.

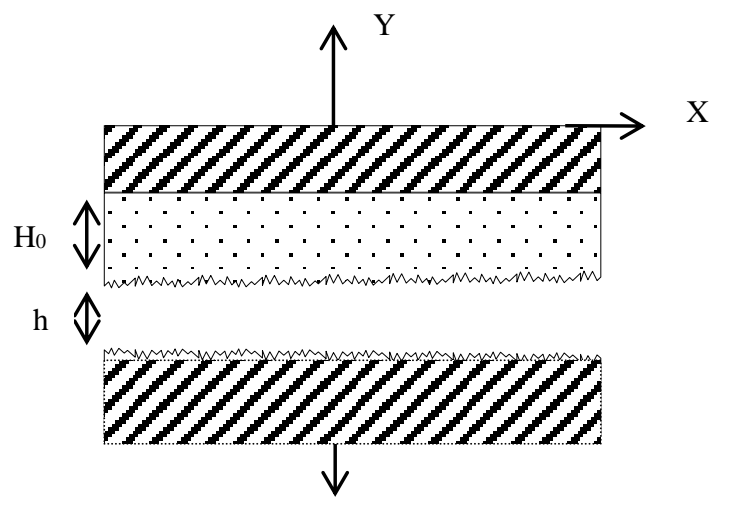

Figure: 1 Configuration of the bearing

The bearing surfaces are considered to be transversely rough. Accordingly, the study [Christensen $\&$ Tonder 1969-70] presents the geometry of local film thickness to be considered as

$$
h(x)=\bar{h}(x)+h_{s}(x)
$$

where, $\bar{h}(x)$ is the mean film thickness and $h_{s}(x)$ is the deviation from the mean film thickness characterizing the random roughness of the bearing surfaces. The details regarding the roughness and characterization can be obtained from [Christensen \& Tonder 1969-70].

Axially symmetry flow of the magnetic fluid between the rectangular plates under an oblique magnetic field is considered. The form of the magnitude $H$ of the magnetic field has been employed from [Acharya et al., 2015] as

$$
H^{2}=k b^{2}\left(\frac{3 \pi \bar{z}}{2}+\frac{3 \pi}{4}\right) \cos \left(\frac{3 \pi \bar{z}}{2}-\frac{\pi}{4}\right)
$$

$k$ being a suitably chosen constant so as to produce a magnetic field of required strength.

Consideration of the stochastic averaging method of [Christensen \& Tonder 1969-70] delivers the Reynolds' type equation for pressure distribution as

$$
\frac{d^{2}}{d z^{2}}\left(p-0.5 \mu_{0} \bar{\mu} H^{2}\right)=\frac{12 \mu \dot{h}}{g_{1}(h)+12 \phi H_{0}}
$$

where

$$
g_{1}(h)=h^{3}+3 \sigma^{2} h+3 h^{2} \alpha+3 h \alpha^{2}+3 \sigma^{2} \alpha+\alpha^{3}+\varepsilon
$$

Now, the effect of deformation [Prajapati, 1995] transforms this equation into 


$$
\frac{d^{2}}{d z^{2}}\left(p-0.5 \mu_{0} \bar{\mu} H^{2}\right)=\frac{12 \mu \dot{h}}{g_{2}(h)}
$$

where,

$$
\begin{aligned}
g_{2}(h) & =\left(h+p_{a} p^{\prime} \delta\right)^{3}+3\left(\sigma^{2}+\alpha^{2}\right)\left(h+p_{a} p^{\prime} \delta\right) \\
& +3\left(h+p_{a} p^{\prime} \delta\right)^{2} \alpha+3 \sigma^{2} \alpha+\alpha^{3}+\varepsilon+12 \phi H_{0}
\end{aligned}
$$

Lastly, the Beavers - Joseph slip model [Beavers \& Joseph, 1967] converts this equation in to the following

$$
\frac{d^{2}}{d z^{2}}\left(p-0.5 \mu_{0} \bar{\mu} H^{2}\right)=\frac{12 \mu \dot{h}}{g(h)}
$$

where,

$$
\begin{gathered}
g(h)=A\left(h+p_{a} p^{\prime} \delta\right)^{3}+3 A^{1 / 3}\left[\left(\sigma^{2}+\alpha^{2}\right)\left(h+p_{a} p^{\prime} \delta\right)+4 \phi H_{0}\right] \\
+3 A^{2 / 3}\left(h+p_{a} p^{\prime} \delta\right)^{2} \alpha+3 \sigma^{2} \alpha+\alpha^{3}+\varepsilon \\
A=\frac{4+s\left(h+p_{a} p^{\prime} \delta\right)}{2+s\left(h+p_{a} p^{\prime} \delta\right)}
\end{gathered}
$$

Integrating above equation (7) in view of the boundary conditions

presents the pressure distribution as

$$
p\left( \pm \frac{b}{2}\right)=0
$$

$$
p=0.5 \mu_{0} \bar{\mu} H^{2}+\frac{6 \mu \dot{h} b^{2}}{g(h)}\left(\frac{z^{2}}{b^{2}}-\frac{1}{4}\right)
$$

Introduction of the non-dimensional quantities

$$
\begin{gathered}
\bar{z}=\frac{z}{b} ; \mu^{*}=-\frac{k h^{3} \mu_{0} \bar{\mu}}{\mu \dot{h}} ; \psi=\frac{\phi H_{0}}{h^{3}} ; \bar{\sigma}=\frac{\sigma}{h} ; \bar{\alpha}=\frac{\alpha}{h} \bar{\varepsilon}=\frac{\varepsilon}{h^{3}} ; \bar{p}=p_{a} p^{\prime} ; \bar{\delta}=\frac{\delta}{h} ; \bar{s}=s h ; \\
P=-\frac{h^{3} p}{\mu \dot{h} b} ; W=-\frac{h^{3} w}{\mu \dot{h} b^{2}}
\end{gathered}
$$

leads to the pressure distribution in dimensionless form as

$$
P=\frac{\mu^{*}}{2}\left(\frac{3 \pi \bar{z}}{2}+\frac{3 \pi}{4}\right) \cos \left(\frac{3 \pi \bar{z}}{2}-\frac{\pi}{4}\right)+\frac{6}{G(\bar{h})}\left(\bar{z}^{2}-\frac{1}{4}\right)
$$

where,

$$
\begin{aligned}
G(\bar{h}) & =\bar{A}(1+\bar{p} \bar{\delta})^{3}+3 \bar{A}^{1 / 3}\left[\left(\bar{\sigma}^{2}+\bar{\alpha}^{2}\right)(1+\bar{p} \bar{\delta})+4 \psi\right] \\
& +3 \bar{A}^{2 / 3}(1+\bar{p} \bar{\delta})^{2} \bar{\alpha}+3 \bar{\sigma}^{2} \bar{\alpha}+\bar{\alpha}^{3}+\bar{\varepsilon} \\
& \bar{A}=\frac{4+\bar{s}(1+\bar{p} \bar{\delta})}{2+\bar{s}(1+\bar{p} \bar{\delta})}
\end{aligned}
$$


The load bearing capacity then, can be expressed in dimensionless form as

$$
W=0.6061 \mu^{*}+[G(\bar{h})]^{-1}
$$

\section{Results and discussions}

Equation (12) shows that the non-dimensional load carrying capacity $\mathrm{W}$ enhances by

$$
0.6061 \mu^{*}
$$

as compared to the case of a conventional lubricant based bearing system. In the lack of deformation and slip this investigation reduces to analysis of [Acharya et al., 2015] and the absence of magnetization leads to the investigation of [Prakash \& Vij, 1973] for smooth bearing systems, when no slip and deformation are involved.

The effect of deformation found in figures (2)-(7) makes it clear that deformation has a significant effect for improving the performance of a bearing system. It is noticed that the load bearing capacity reduces considerably owing to the deformation. The effect of magnetization shown in figures (8)-(10) establishes that $\mathrm{W}$ increases significantly with an increase in the magnetization parameter.

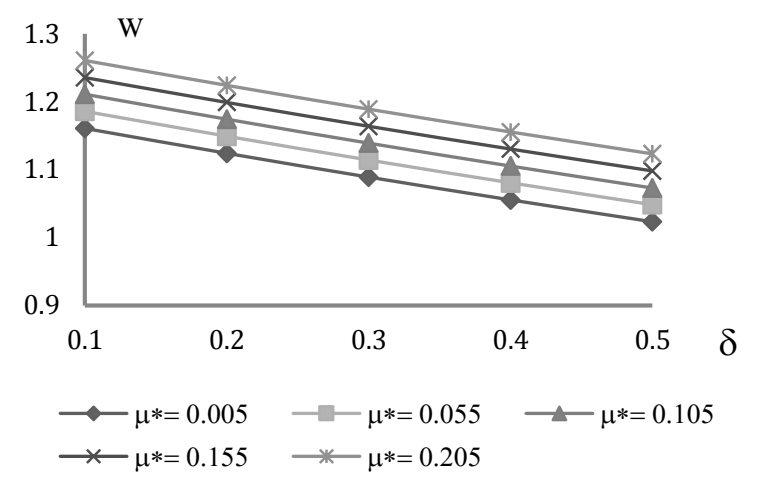

Figure: 2 Variation of W with respect to $\bar{\delta}$ and $\mu^{*}$

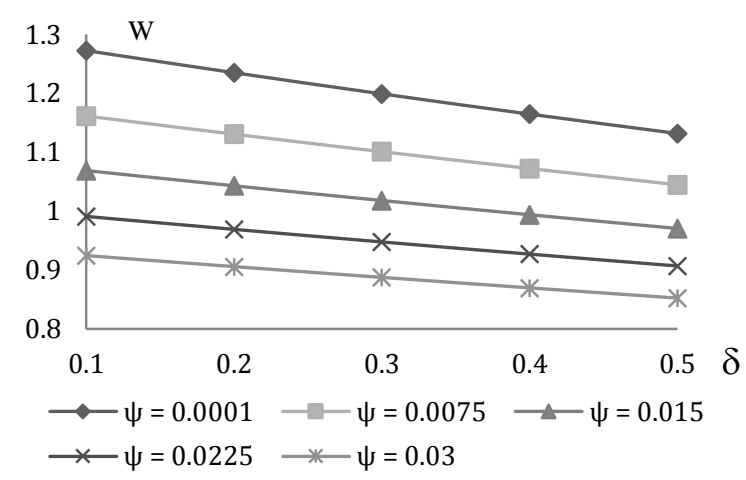

Figure: 3 Variation of $\mathrm{W}$ with respect to $\bar{\delta}$ and $\psi$ 


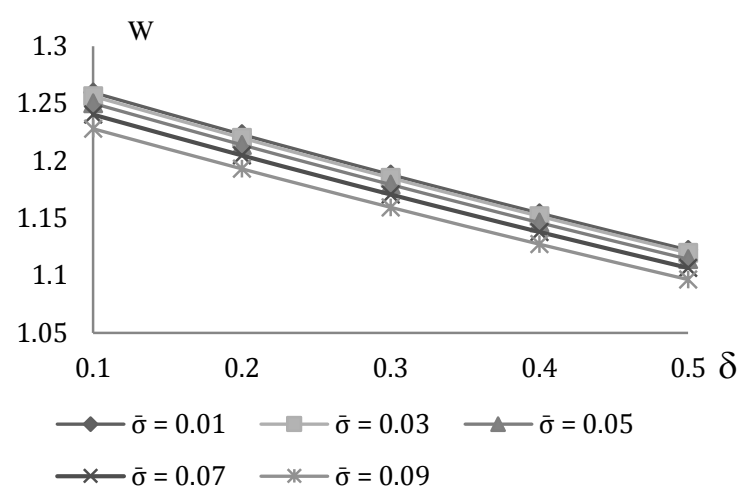

Figure: 4 Variation of $\mathrm{W}$ with respect to $\bar{\delta}$ and $\bar{\sigma}$

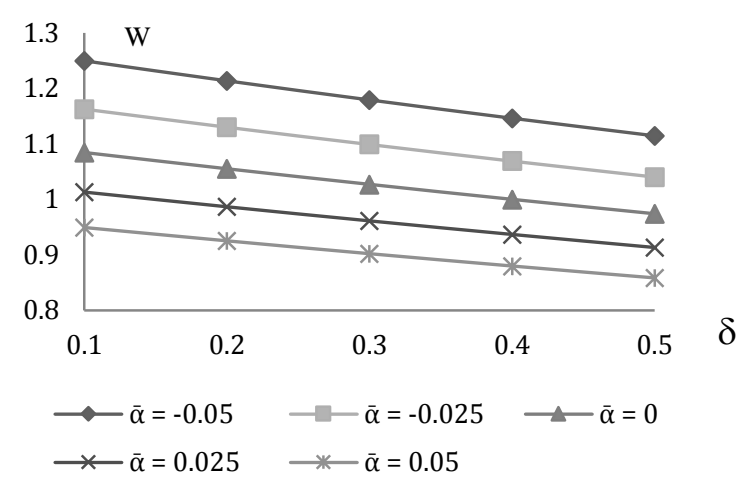

Figure: 5 Variation of W with respect to $\bar{\delta}$ and $\bar{\alpha}$

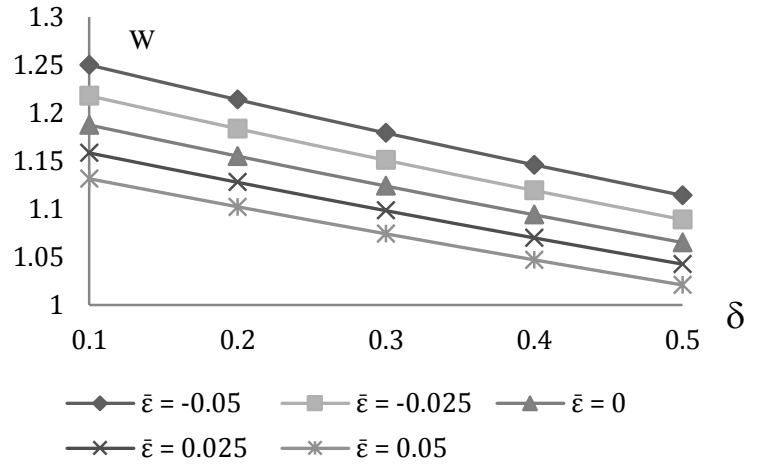

Figure: 6 Variation of W with respect to $\bar{\delta}$ and $\bar{\varepsilon}$ 


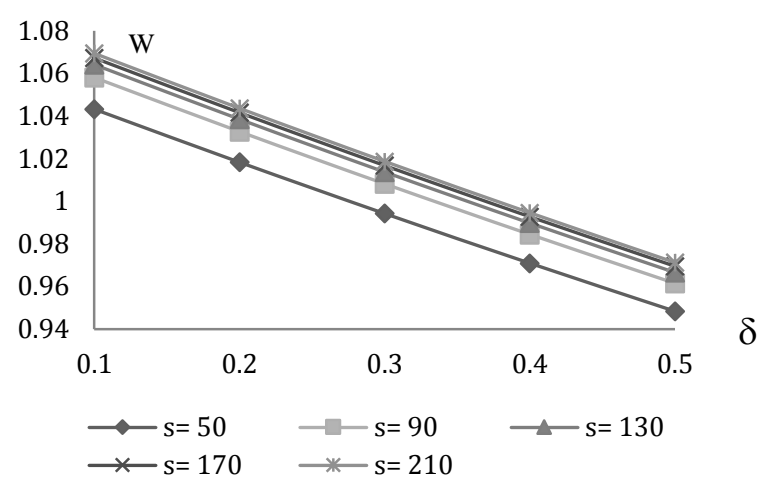

Figure: 7 Variation of W with respect to $\bar{\delta}$ and $\bar{s}$

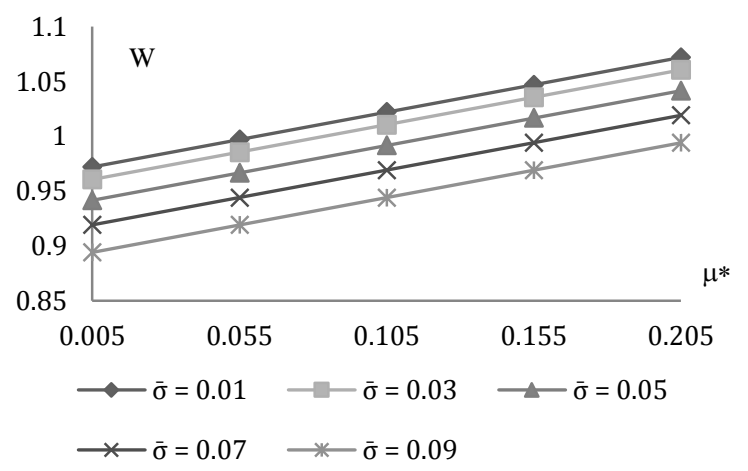

Figure: 8 Variation of $\mathrm{W}$ with respect to $\mu^{*}$ and $\bar{\sigma}$

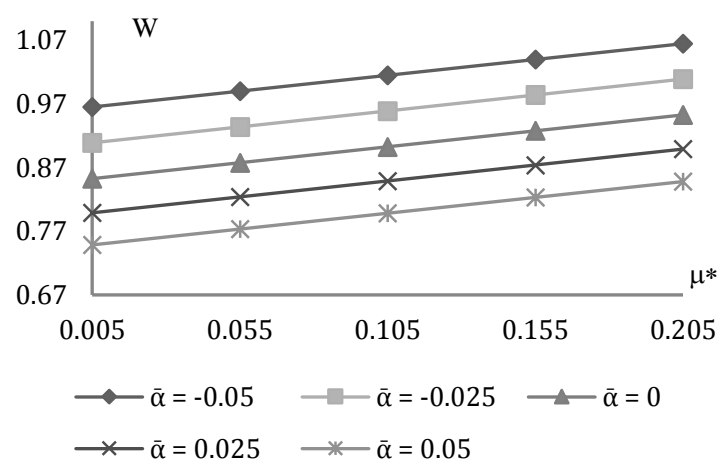

Figure: 9 Variation of $\mathrm{W}$ with respect to $\mu^{*}$ and $\bar{\alpha}$ 


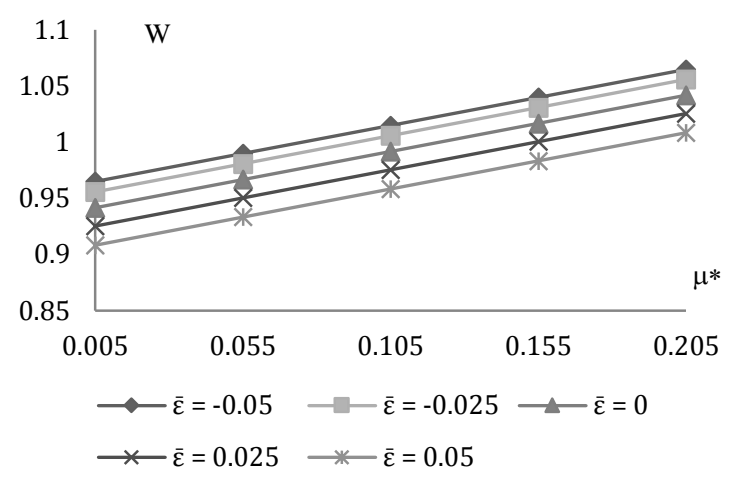

Figure: 10 Variation of W with respect to $\mu^{*}$ and $\bar{\varepsilon}$

The effect of porosity is to bring down the $\mathrm{W}$ as can be seen from figures (11)-(13). Further, moderate to large values of slip aggravates the situation. The standard deviation associated with the roughness turns in decreased W [figures (3) and (7)]. As expected, the slip effect turns in an adverse performance [figures (14)-(15)]. Besides, the negatively skewed roughness gives rise to increased load. In addition, the trends of $\mathrm{W}$ with respect to variance are almost alike to that of skewness.

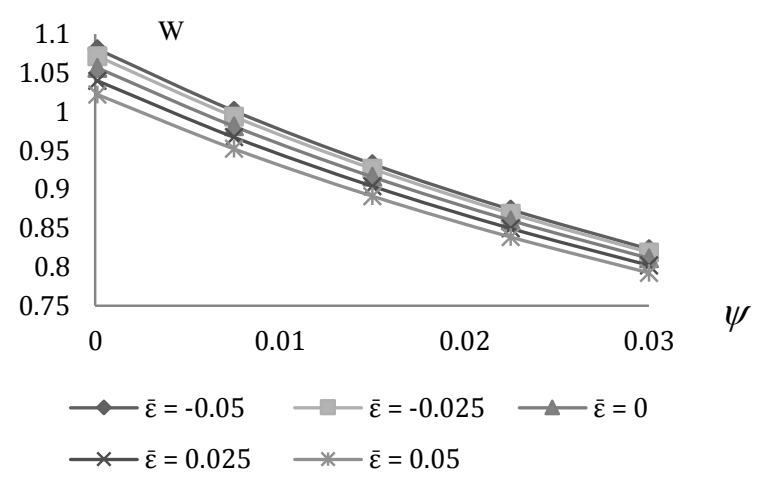

Figure: 11 Variation of $\mathrm{W}$ with respect to $\psi$ and $\bar{\varepsilon}$

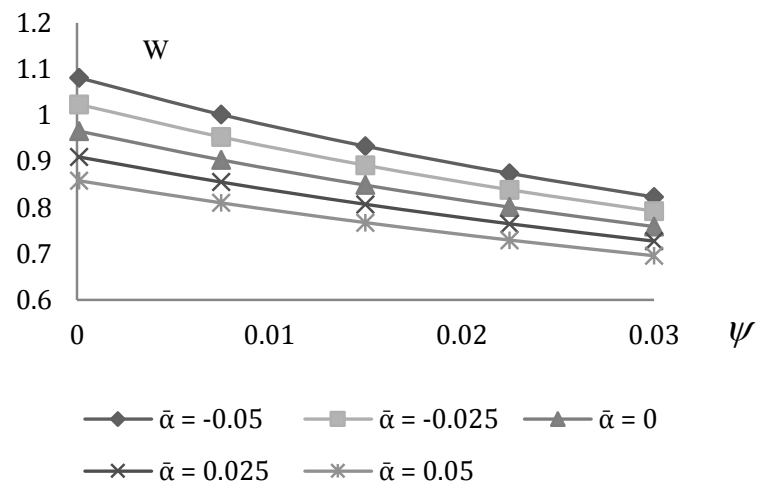

Figure: 12 Variation of $\mathrm{W}$ with respect to $\psi$ and $\bar{\alpha}$ 


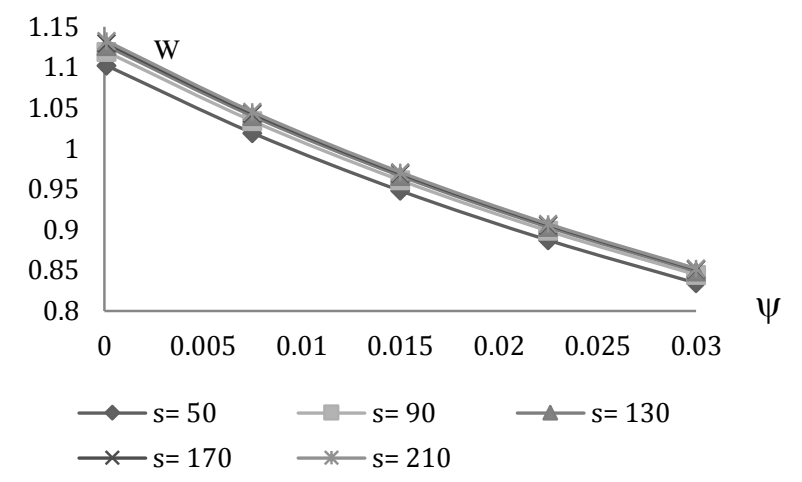

Figure: 13 Variation of W with respect to $\psi$ and $\bar{S}$

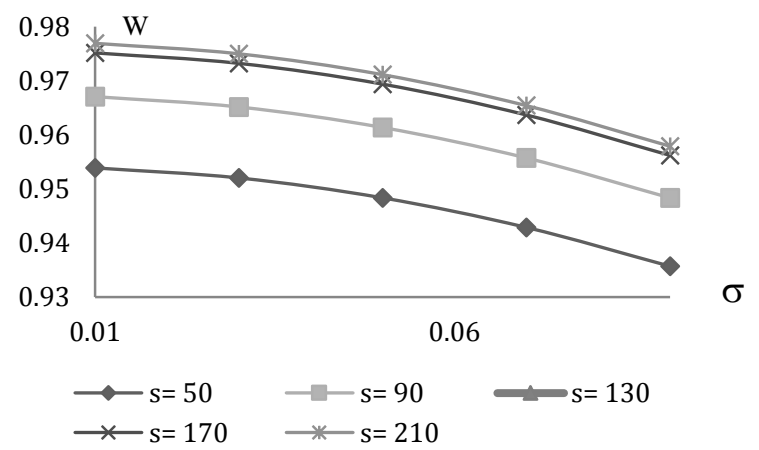

Figure: 14 Variation of W with respect to $\bar{\sigma}$ and $\bar{s}$

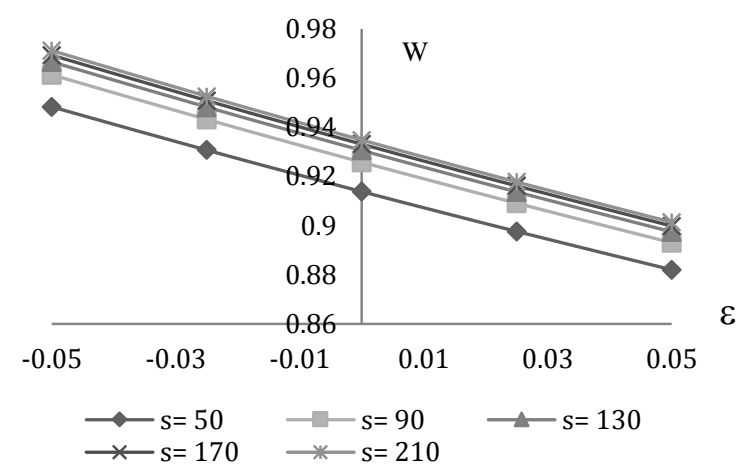

Figure: 15 Variation of W with respect to $\bar{\varepsilon}$ and $\bar{s}$

In addition, the following points are observed:

1. The combined positive effect of negatively skewed roughness and variance (- ve) may be provide a better option towards the improvement of bearing performance ewhen the slip is 
at minimum level. This effect further advances when small values of deformation are involved.

2. Even if, the slip parameter is at reduced level, the porosity- deformation combine results in significant load reduction.

3. For a large range of deformation parameter, the duo of slip velocity and standard deviation affects the bearing performance significantly.

\section{Conclusion}

The trio of deformation, slip and porosity induces significant load reduction. The situation further worsens when large values of standard deviation are involved. Although, the $\mathrm{W}$ increases sharply due to magnetization, the magnetic fluid lubrication has a limited option in minimizing the above adverse effect. However, the circumstances remain more favorable in the case of negatively skewed roughness. This indicates that the roughness remains a crucial aspect to be addressed for designing this type of bearing systems.

Even if one considers lower values of slip and deformation, the magnetic fluid lubrication offers a narrow option in countering the adverse effect of porosity and standard deviation. The absence of flow fails to deter the bearing system from supporting a good amount of load that remains far away from being true in the case of conventional fluid based bearing system.

Having the model developed accentuating the wear and temperature raise aspect; it can serve in censors and shock absorbers.

\section{Nomenclature}

\begin{tabular}{|c|l|c|l|}
\hline Symbol & Name & Symbol & Name \\
\hline$p$ & Lubricant pressure & $\mu_{0}$ & The permeability of free space \\
\hline$w$ & Load carrying capacity & $P$ & Dimensionless pressure \\
\hline$\alpha$ & Variance & $W$ & Dimensionless load carrying capacity \\
\hline$\sigma$ & Standard deviation & $\bar{\alpha}$ & Variance in non-dimensional form \\
\hline$\varepsilon$ & Skewness & $\bar{\sigma}$ & Dimensionless standard deviation \\
\hline$\mu$ & Viscosity of lubricant & $\bar{\varepsilon}$ & Non-dimensional skewness \\
\hline$\delta$ & Deformation & $\mu^{*}$ & $\begin{array}{l}\text { Dimensionless magnetization } \\
\text { parameter }\end{array}$ \\
\hline$p_{a}$ & The reference ambient pressure & $\bar{\delta}$ & Dimensionless deformation \\
\hline
\end{tabular}

\section{Acknowledgement}

The authors are very much thankful to the reviewers for their valuable and fruitful suggestions. 


\section{References}

Beavers, G. S and Joseph, D. D (1967). Boundary conditions at a naturally permeable wall, Journal of fluid mechanics, (vol. 30, pp. 197-207).

Cameron A (1966). The Principles of Lubrication. Longmans, London.

Tzeng, S. T. and Saibel, E(1967). Surface roughness effect on slider bearing lubrication. ASLE Transaction, (vol.10, pp. 334-338).

Christensen, H., Tonder, K., (1969a).Tribology of rough surfaces, Stochastic models of hydrodynamic lubrication, SINTEF, Section for Machine Dynamics in Tribology, Technical University of Norway, Trondheinm, Norway, Report No.10/69-18.

Christensen, H. and Tonder, K(1969b).Tribology of rough surfaces, parametric study and comparison of lubrication models, SINTEF, Section for Machine Dynamics in Tribology, Technical University of Norway, Trondheinm, Norway, Report No.22/69-18.

Christensen, H. and Tonder, K(1970).The hydrodynamic lubrication of rough bearing surfaces of finite width, ASME-ASLE Lubrication conference Cincinnati, Ohio, October 12-15, Lub-7.

Christensen, H and Prakash, J (1978). Squeeze films between two rough rectangular plates, Journal of mechanical engineering science, (vol. 20, pp.183).

Guha, S. K (1993).Analysis of dynamic characteristic of hydrodynamic journal bearings with isotropic roughness effects, Wear, (vol. 167(1), pp. 173-180).

Bhat M. V. and Deheri G. M (1993). Squeeze film behaviour in porous annular disks lubricated with magnetic fluid, Wear, (vol.151, pp. 123-128).

Gupta, J. L. and Deheri, G. M. (1996). Effect of roughness on the behaviour of squeeze film in a spherical bearing, Tribology Transactions, (vol. 39, pp. 99-102).

Prakash, J. and Gururajan, K. (1999). Effect of velocity slip in an infinitely long rough porous journal bearing, Tribology Transactions, (vol. 42(3), pp. 661-667).

Patel, R. M., Deheri, G. M. and Vadher, P. A. (2014).Performance of a magnetic fluid based squeeze film between infinitely long rough porous rectangular plates, Proceeding of ICATES, DOI: 10. 1007/978-81-322-1656-8_5. Springer India.

Shimpi, M. E. and Deheri, G. M. (2014). Surface roughness effect on a magnetic fluid-based squeeze film between a curved porous circular plate and a flat circular plate, Journal of the Brazilian society of mechanical sciences and engineering (Springer), (vol. 36(2), pp. 233-243).

Patel, R. M., Deheri, G. M. and Vadher, P. A. (2014).Magnetic fluid based squeeze film in rough porous long plates: Effect of non-uniform thickness, SOP Transaction on applied mathematics, (vol. 1(2), pp.194-209).

Murti, P. R. K. (1973).Effect of slip-flow in narrow porous bearings, Journal of Lubrication Technology, (vol. 95 (4), pp. 518-523).

Verma, P. D. S. (1986).Magnetic fluid based squeeze films, International journal of engineering sciences, (vol. 24(3), pp. 395-401).

Agrawal, V.K. (1986).Magnetic fluid based porous inclined slider bearing, Wear,(vol. 107, pp. 133-139).

Fortier Alicia, E. and Salant Richard, F. (2005).Numerical analysis of journal bearing with heterogeneous slip/no-slip surfaces, Journal of Tribology, (vol. 27 (4), pp. 820-825).

Shukla, S. D. and Deheri, G. M. (2014).Effect of slip velocity on the performance of a magnetic fluid based squeeze film in porous rough infinitely long parallel plates, Friction and wear research, (vol. 2(1), pp. 6-16).

Higginson, G. R. (1965).The theoretical effects of elastic deformation on the bearing liner on Journal bearing performance, Proceedings of the Institution of Mechanical Engineers, Conference Proceedings June, (vol. 180(2), pp. 31-38). 
Prakash, J. and Peeken, H. (1985).The combined effect of surface roughness and elastic deformation in the hydrodynamic slider bearing problem, ASLE Transactions, (vol. 28(1), pp. 6974).

Schwarz, U. B. (2003).A generalized analytical model for the elastic deformation of an adhesive contact between a sphere and a flat surface, Journal of Colloid and Interface Science, (vol. 261(1), pp. 99-106).

Chatchai, A. and Mongkol, M. (2010).Influence of surface roughness on elastohydrodynamic journal bearings with non-Newtonian lubricants, Advanced Tribology, (Part 3, pp. 50-51).

Shimpi, M. E., and Deheri, G. M. (2013).Ferrofluid lubrication of rotating curved rough porous circular plates and effect of bearing's deformation, Arabian journal for science and engineering (Springer), (vol.38 (10), pp. 2865-2874).

Acharya, A. S., Patel R. M. and Deheri, G. M. (2015).Ferro fluid squeeze film in infinitely long porous rough rectangular plates, International journal of scientific and engineering research, (vol. 6 (8)).

Prajapati, B. L. (1995).On certain theoretical studies in hydrodynamic and electromagneto hydrodynamic lubrication. Ph.D. Thesis, Sardar Patel University. Vallabh Vidyanagar, Anand, Gujarat, India.

Prakash J. and Vij S.K. (1973).Load capacity and time height relations for squeeze film between porous plates, Wear, (vol. 24, pp. 309-322). 